\title{
Posiciones de organizaciones defensoras de derechos humanos ante medidas migratorias australianas recientes y su impacto en la política migratoria
}

\author{
Wendy Mosquera Bonilla ${ }^{1}$ \\ wembonilla@gmail.com
}

Reporte de caso recibido el 30/09/2010 y aprobado el 11/03/2011

\begin{abstract}
Resumen
La política migratoria australiana en los últimos años ha sido abierta, pero controlada.Durante la última década,Australia ha recibido una gran cantidad de población migrante calificada a pesar de sus precarias condiciones geográficas y climáticas. Sin embargo,las políticas implementadas para los solicitantes de asilo han sido bastantes represivas y durante los primero meses del año, debido al aumento de arribos en embarcaciones ilegales de indocumentados, se han recrudecido. Esta situación ha generado estupor en organizaciones defensoras de derechos humanos, y por tal razón este corto documento busca enmarcar las discusiones surgidas a partir de ese hecho expuestas por diferentes medios escritos y el impacto de esas posiciones en la construcción de las políticas para inmigrantes.
\end{abstract}

Palabras clave: Asilo, ACNUR, Australia, refugiados, política migratoria.

\begin{abstract}
Australian immigration policy in recent years has been open but controlled. During the last decade, Australia has received a large number of skilled migrants despite their precarious geographical and climatic conditions. However, the policies implemented for asylum seekers have been repressive, and during the first months of the year, due to increased illegal boat arrivals with illegal immigrants, have worsened.This situation has caused an emphatic reaction from the human rights organizations, and for that reason, this paper seeks to frame those discussions arising from the fact that has been set out by the media and the impact of these positions in the construction of policies for immigrants.
\end{abstract}

Key Words: Asylum, ACNUR, Australia, refugees, immigration policy.

1 Wendy Bonilla es Comunicadora social y periodista de la Universidad del Valle y candidata al título de politóloga en la Universidad Icesi 


\section{Introducción}

Decepcionante. Esta fue la palabra usada por la Agencia de las Naciones Unidas para los Refugiados,ACNUR desde su sede regional en Canberra capital de Australia, para explicar su desacuerdo por el "fracaso para tomar alternativas a la detención de solicitantes de asilo que llegan en botes sin problemas de salud o riesgo al público". Aunque la preocupación no se basa únicamente en la detención de personas en bases militares ubicadas en Christmas Island y Darwin, sino también en la "suspensión de forma inmediata de todos los procesos abiertos para la concesión de asilo a inmigrantes procedentes de Afganistán y Sri Lanka como parte de una campaña para luchar contra las bandas que se lucran con el tráfico de personas". ${ }^{3}$ Esa inquietud no solamente la tiene la Agencia, sino varias de las organizaciones de defensa de derechos humanos con sede en el país.

Tomando como referencia este complejo escenario,el presente escrito pretende abordar las posiciones de organizaciones defensoras de los derechos humanos, reportadas en medios escritos durante el mes de abril de 2010, con respecto a las medidas represivas tomadas por el gobierno australiano contra los solicitantes de asilo afganos y ceilandeses, y el impacto

2 UNHCR disappointed at Australian decision to reopen detention centre for asylum-seekers [en línea]. The UN Refugee Center UNHCR [consultado 17 de abril,2010].Disponible en Internet: http://www.unhcr. org/4bcd99956.html

3 Australia suspende las concesiones de asilo a afganos y ceilandeses para evitar el tráfico de personas [en línea]. Europa Press [consultada 2 de mayo, 2010] Disponible en Internet: http://www.europapress.es/ epsocial/politica-social/noticia-australia-suspendeconcesiones-asilo-afganos-ceilandeses-evitar-traficopersonas-20100409080111.html de esas sobre el modo como se ha venido construyendo la política migratoria en Australia.

La decisión de suspender los procesos de visado para solicitantes de asilo provenientes de Afganistán y Sri Lanka fue tomada el 08 de abril de 2010 por el primer ministro australiano Kevin Rudd, quien de acuerdo a la prensa de ese país actuó bajo presión de la oposición, liderada por Tony Abbott lo que representa un "retroceso vergonzoso en su política de inmigración blanda". ${ }^{4}$

Fue en 2008 cuando el nuevo gobierno laborista de Kevin Rudd abandonó la controversial política de su antecesor de no encarcelar a los inmigrantes solicitantes de asilo que lleguen en bote a las costas australianas; y la abolición de las Visas Temporales Humanitarias y de Protección por visas de tipo permanentes, en donde aquellos solicitantes que se les "conceda el estatus de refugiado tendrán protección permanente, en lugar de tener que reaplicar cada tres años por su visa [...] vivirán con seguridad de residencia en Australia y podrán aplicar para la reunión con miembros de sus familias que no han visto". ${ }^{5}$ En esa época la decisión fue alababa por diversas ONG como Amnistía Internacional.Sin embargo, muchos críticos afirman que debido a esto, los arribos de inmigrantes en botes han aumentado, en especial provenientes de Afganistán y

4 PM's soft boat policy links [en línea]. The Herald Sun [consultado 1 de mayo, 2010]. Disponible en Internet: http://www.heraldsun.com.au/opinion/ editorials/pms-soft-boat-policy-sinks/story-e6frfhqo-1225852003403

5 Australia's Refugee and Humanitarian Program: Community views on current challenges and future directions. Refugee Council of Australia, 2009.81 p. 
Sri Lanka, representando un " $80 \%$ de los arribos en los últimos 16 meses". ${ }^{6}$ Estos dos países son políticamente inestables lo que hace que la vida de sus ciudadanos sea demasiado frágil en sus lugares de origen $y$, posiblemente, no estén lo suficientemente preparados para afrontar los retos que implica el llegar a una nueva nación. Es decir que, en el caso tal de que el Gobierno australiano no tome medidas de política pública en los asuntos migratorios, es de esperar que los arribos de migrantes impliquen consecuencias considerablemente negativas para el pueblo australiano. A modo de ejemplo, en Afganistán

los derechos humanos no se respetan debido a la continua insurgencia, gobierno e instituciones débiles, corrupción, tráfico de drogas y el legado de tres décadas de conflicto [...] se cometieron asesinatos extrajudiciales, torturas, restricciones en libertadas de religión, movimiento y asociaciones, violencia y discriminación contra las mujeres, minorías religiosas, tráfico de personas y explotación infantil. ${ }^{7}$

En cuanto a Sri Lanka, a pesar que la guerra civil hubiese terminado el año 2009,la situación de las minorías étnicas es aun precaria. Por esto son cada vez más las personas de estas nacionalidades que emigran a las costas australianas en embarcaciones pesqueras y en condiciones infrahumanas. Esta situación, para las

6 Tony Abbott mocks government suspension of asylum seeker claims [en línea].The Herald Sun [consultado 1 de mayo,2010].Disponible en Internet: http:// www.heraldsun.com.au/news/national/tony-abbottmocks-government-suspension-of-asylum-seekerclaims/story-e6frf7l6-1225852025577

7 Australia's Refugee and Humanitarian Program: Community views on current challenges and future directions. Refugee Council of Australia, 2009.97 p. autoridades de ese país representa un riesgo para la seguridad de los inmigrantes, debido a la existencia de bandas dedicadas a traficar con ellos. De acuerdo a reportes de la ACNUR en lo que va corrido del año han llegado cerca de 1800 personas en busca de asilo.

Las Naciones Unidas define como refugiado a "cualquier persona que debido al temor fundado de ser perseguido por razones de raza, religión, nacionalidad o membresía a un grupo social o político en particular, se encuentra fuera de su país de nacionalidad y es incapaz o debido a ese temor, no se puede beneficiar de la protección de su país". ${ }^{8}$ En el mundo, hay 9.8 millones de refugiados, 1.71 millones de personas tienen condiciones de refugiados, 740 mil son solicitantes de asilo. ${ }^{9}$

De esta forma, personas que sean considerados bajo estos estándares pueden aplicar a visas de asilo en diferentes países ya sean industrializados o en vía de desarrollo, inclusive la mayoría de las poblaciones de refugiados y personas en condiciones de refugiados se encuentran en países pobres. De acuerdo a datos suministrados por el Consejo de Refugiados en Australia,en la actualidad este país ha ofrecido alojamiento a menos del $1 \%$ de la población desplazada. Sin embargo, para el 2008 entre los países industrializados que atienden peticiones de refugiados,

\footnotetext{
8 Refugee Convention,Article 1A(2), Protocol,Art1(A) (2). Citado por: Refugee and Humanitarian Program. Asa Group [consultado 1 de mayo,2010]. Disponible en Internet: http://www.australia-migration.com/page/ Refugee_and_Humanitarian_Program/34

9 Australia's Refugee and Humanitarian Program: Community views on current challenges and future directions. Refugee Council of Australia, 2009.18 p.
} 
ocupaba el puesto número seis, lo cual de acuerdo al estudio, se presenta por la geografía del continente que hace que menos personas soliciten asilo.

\section{Refugiados y migración internacional en Australia}

En las últimas décadas, Australia ha tenido una política de apertura a la inmigración controlada de personas en edad productiva que puedan insertarse y asimilar la cultura de ese país para generar desarrollo dentro del mismo. Ha abierto sus fronteras para el ingreso de personas de todo el mundo sin importar la cantidad y los efectos que puede tener esa magnitud debido a sus condiciones geográficas y ambientales. Durante varios años han establecido políticas de inclusión para los extranjeros, pero no cualquier extranjero, sino los calificados, profesionales, menores de 45 años y con buen nivel del idioma inglés.

El gobierno australiano tiene tres programas principales: el primero, 'Migración familiar', en el cual, un inmigrante residente en Australia puede solicitar visas de residente para sus familiares más cercanos. El segundo, 'Skill migration' que a su vez está dividido en cinco categorías, el objetivo de este programa está en ayudar en el proceso de migración a profesionales o mano de obra calificada. El tercero, y quizá el que más ha llamado la atención de defensores humanos, el 'Humanitarian program' (Programa Humanitario) que busca conceder asilo a personas perseguidas, desplazadas o que se les están vulnerando los derechos fundamentales.

El Programa Humanitario, tiene dos componentes básicos: el primero,'Onsho- re Protection/ Asylum Component',"ofrece protección a personas que ya se encuentran en Australia y que son reconocidos como refugiados”. ${ }^{10} \mathrm{El}$ segundo, 'Offshore Resettlement Component', "brinda hospedaje a extranjeros para quienes esta es la opción más apropiada". ${ }^{11}$ Este último componente que está dividido en dos categorías: Refugiado y Programa Humanitario Especial ofrece visas permanentes; mientras que el primer componente hasta hace dos años ofrecía visas temporales. Situación que cambió en el gobierno de Kevin Rudd y que hoy está en la palestra pública debido a la suspensión de asilos.

Para organizaciones como el Consejo de Refugiados en Australia, el aporte de dicho país en cuestiones de concesión de visas humanitarias es muy bajo, en comparación con otras naciones, lo que lo lleva a ocupar el puesto número 41 de acuerdo al ranking de la ACNUR, en 2007. Los datos suministrados por el Departamento de Inmigración y Ciudadanía, indica que en total en 2008, 13.502 visas fueron otorgadas de las cuales 6.499 pertenecen al programa de refugiados, 4.625 al especial humanitario y 2.378 al de asilo

\section{Debate migratorio}

A pesar de que en Australia aún existen políticas fuertes y cuestionables contra la inmigración como la detención de solicitantes de asilo en lugares alejados como bases militares - política que hace algún tiempo había sido abolida - este, es un país que en los últimos años ha mostrado

10 National Communications Branch, Department of Immigration and Citizenship, Canberra. Fact Sheet 60 - Australia's Refugee and Humanitarian Program [en línea] [Consultado 1 de mayo,2010] Disponible en Internet: www.immi.gov.au

11 Ibid. 
apertura de fronteras a trabajadores calificados y profesionales de diversas áreas. En este sentido, desde hace más de diez años los partidos mayoritarios han congeniado en sus políticas de inmigración. Esto se puede ver en el discurso oficial del gobierno, en el cual se exaltan los efectos positivos de la inmigración en la economía australiana, como la "[...] expansión de los negocios [...] diversidad productiva a través del conocimiento de los mercados internacionales [...] capital intelectual y mano de obra novedosa". ${ }^{12}$

Sin embargo, hay sectores como los grupos ambientalistas que se encuentran en desacuerdo sobre todo por las magnitudes en la inmigración, en la medida en que migraciones a gran escala a un país con condiciones climáticas tan precarias podría ser desventajoso.

Por su parte,el partido Demócrata Australiano está completamente en desacuerdo con la apertura de fronteras a grupos de extranjeros y hay investigaciones que indican que esto se debe a que aún hay quienes tienen el deseo de una Australia homogénea y "la hostilidad frente a grupos no - europeos aún existe". ${ }^{13}$

Con respecto al programa humanitario, que es el que más debate suscitó sobre todo durante el mes de abril de 2010, en donde debido al ablandamiento de las políticas de detención y visados permanentes en lugar de temporales, la prensa

12 National Communications Branch, Department of Immigration and Citizenship, Canberra. Fact Sheet 4 - More than 60 Years of Post-war Migration [en línea] [Consultado 23 de febrero,2010] Disponible en Internet: www.immi.gov.au

13 Asia Pacific Migration Research Network (APMRN). Migration issues in the Asia Pacific: Issues paper from Australia [en línea]. [Consultado 13 de febrero, 2010] Disponible en Internet: http://www.unesco.org/most/ apmrnwp5.htm australiana reportó que hasta el mes de mayo habían llegado 38 embarcaciones con inmigrantes indocumentados, la última fue rescatada por la marina australiana y llevaba 70 personas desplazadas.

Sin embargo, las organizaciones defensoras de los derechos humanos no han dudado en manifestarse por la situación humanitaria de los solicitantes de asilo. La primera en manifestarse, como se explicó anteriormente fue la Agencia de las Naciones Unidas para los Refugiados, que calificó las medidas represivas contra los refugiados que son llevados a bases militares en lugares lejanos y la suspensión de peticiones de visa, como 'decepcionante'. La Agencia indicó en un comunicado, que la "experiencia ha demostrado que estas medidas pueden tener un impacto negativo en la salud y en el bienestar de los afectados por los últimos anuncios, especialmente aquellos que ya han sufrido tortura o experiencias traumáticas antes de llegar a este país". ${ }^{14}$ Sin embargo, el papel de las Naciones Unidas ha ido más allá de comunicados expresando desacuerdo ante las políticas migratorias, en la medida que continúa entablando "conversaciones con el gobierno para saber cómo minimizar el impacto de la suspensión de solicitudes de asilo y el impacto de la detención obligatoria en la salud y el bienestar de los desplazados". ${ }^{15}$

Esto hace pensar que la agenda de este tipo de organizaciones sigue enfocada en ayudar a solucionar las dificultades de los grupos vulnerables. Aunque el compro-

14 UNHCR disappointed at Australian decision to reopen detention centre for asylum-seekers [en línea]. The UN Refugee Center UNHCR [consultado 17 de abril,2010].Disponible en Internet: http://www.unhcr. org/4bcd99956.html

15 Ibid. 
miso de otros sectores no es el mismo. $\mathrm{O}$ por el contrario,y dados los principios de no intervención en los asuntos internos, no se puede hacer nada más que solicitar y comunicar indignación ante una situación como esta. Amnistía Internacional, por ejemplo, que ha sido una de las organizaciones con posiciones más críticas y duras contra la política australiana reciente, indicó que la medida "es inconsistente con las obligaciones internacionales australianas con respecto a la Convención de las Naciones Unidas para los Refugiados de 1951". ${ }^{16}$

Asimismo, Amnistía Internacional, sostuvo que la decisión es "atroz [...] y pasa por encima de los derechos de los más frágiles [...] y envía un peligroso mensaje hacia la región pacífico asiática". ${ }^{17}$ Esto último, de acuerdo a la Agencia tiene que ver con su preocupación de que gobiernos en la región pueden empezar a presionar a la ACNUR para que cese de enviar afganos y ceilandeses a países como Malasia e Indonesia.Con respecto a la información que maneja el gobierno australiano,y con la cual arguyen que la situación política en Afganistán y Sri Lanka no es tan precaria como antes, Amnistía dice que muchos grupos en Sri Lanka, como activistas, periodistas yTamiles aún se encuentran en peligro. De igual forma indican que muchos en Afganistán, como mujeres cabeza de hogar, huérfanos, gente que requiere atención médica y con discapacidades, están aún en riesgo.

16 Australia asylum suspension could harm world's most vulnerable [en línea]. Amnesty International [consultado 1 de mayo, 2010] Disponible en Internet: http://www.amnesty.org/en/news-and-updates/ australia-asylum-suspension-could-harm-worldsmost-vulnerable-2010-04-09

17 Ibid.
Entre tanto, el Refugee Action Coalition en Sydney expresa que la decisión obedece a la agenda política doméstica y no se basa en las consideraciones de derechos humanos, por lo que demanda retroceder en el congelamiento de las peticiones de asilo de los nacionales ceilandeses y afganos. El Consejo de Refugiados en Australia por su parte, pudo no solamente solicitar revisión en las políticas tomadas, sino que también recordó a ese país es importante mantener la madurez y el equilibrio en el debate sobre los solicitantes de asilo, y también recalca que el compromiso por atender necesidades de personas vulnerables en el mundo no ha sido suficiente.

Para esto, señala que es necesario entender que así como Australia se basa en las estadísticas que inducen el incremento de 1400 solicitudes de asilo el año pasado para proponer una política represiva,ellos también utilizan las estadísticas para explicar lo injustificado de su nueva normativa en tanto que en "Canadá se presentaron 33.250 solicitudes, mientras que en un país pobre como Yemen recibió entre 60 mil y 70 mil arribos en botes y fueron recibidos 4.600 en los últimos 18 meses". ${ }^{18}$ De igual forma, el año pasado, Australia recibió "6170 aplicaciones, siendo esto el $1.6 \%$ de las 377.160 aplicaciones recibidas en 44 naciones industrializadas, lo que ubica a Australia en el puesto $16 "{ }^{19}$ Esto indica que de acuerdo al informe que la misma agencia en 2008 ubicó a este país

18 Halt in refugee processing creates detention concerns [en línea]. The Refugee Council of Australia [consultado 3 de mayo, 2010]. Disponible en Internet: http://www.refugeecouncil.org.au/docs/releases/2010/100418\%20reopen\%20curtin\%20a\%20step\%20 backwards.pdf

19 Ibid. 
en el puesto 6 de aplicaciones recibidas en países industrializados, para el 2009 había perdido aún más posiciones, lo que muestra que tramitó menos solicitudes de asilo de personas vulnerables que muchos de los países industrializados.

De igual forma, el Partido Verde australiano ha manifestado, su inconformidad con la normativa y argumenta que "el primer ministro que ofreció humanidad [...] ahora [propone una] política [que] no tiene nada que ver con dignidad humana", [...] lo que él ha mostrado es cobardía en términos de liderazgo". ${ }^{20}$ Hay que considerar que este partido se ha mostrado en desacuerdo a la migración masiva por cuestiones ambientales, sin embargo, sí apoyan la de tipo humanitaria.

\section{Conclusiones}

El papel que deben tomar las organizaciones defensoras de derechos humanos es la de, además de instar en la evaluación de las medidas ya tomadas, ayudar a mejorar el diálogo entre los refugiados y el gobierno, como lo ha hecho la ACNUR y así agilizar el proceso de visado de indocumentados.

La tarea, al parecer,ya se tienen clara. El gobierno y las ONG deben procurar que los migrantes en situación de vulnerabilidad no sobrelleven el trauma de un conflicto más, ahora en territorio ajeno al de ellos.Las ONG, tienen sus ojos puestos en Australia y, aunque no parece avanzar a una solución pronta de los trámites de los refugiados, el compromiso permanece.

20 Tony Abbott mocks government suspension of asylum seeker claims [en línea].The Herald Sun [consultado 1 de mayo,2010]. Disponible en Internet: http:// www.heraldsun.com.au/news/national/tony-abbottmocks-government-suspension-of-asylum-seekerclaims/story-e6frf7l6-1225852025577
Por su parte,Australia tiene una política migratoria abierta para las personas calificadas, sin embargo, el caso es diametralmente diferente para los migrantes por cuestiones humanitarias. Es deseable que el Gobierno australiano se preocupe por incrementar el número de visas por motivos humanitarias. En este aspecto las ONG deberían tener un rol más proactivo en la promoción de esas normativas, actuando bajo los preceptos de no interferencia en los asuntos internos,más aun cuando son migraciones masivas, que de alguna manera pueden llegar a representar un riesgo para la seguridad del país de acogida, según lo indican las agencias nacionales.

Asimismo es importante pensar que así como hay casos exitosos de inmigrantes calificados, la experiencia cuenta que también los hay de inmigrantes por razones humanitarias porque los "730 refugiados establecidos en Australia desde la Federación han tenido un impacto profundo en la vida social, cultural y económica de la nación [...] sus habilidades, energía, innovación, amor por la libertad y diversidad han enriquecido el país, aumentando su diversidad y adaptándola al cambio del ambiente global [...] son muchas las historias de éxito y contribución". ${ }^{21}$

\section{Bibliografía}

UNHCR disappointed at Australian decision to reopen detention centre for asylumseekers [en línea].The UN Refugee Center UNHCR [consultado 17 de abril, 2010]. Dis-

21 Australia's Refugee and Humanitarian Program: Community views on current challenges and future directions. Refugee Council of Australia, 2009.16 p. 
ponible en Internet: http://www.unhcr. org/4bcd99956.html

Australia suspende las concesiones de asilo a afganos y ceilandeses para evitar el tráfico de personas [en línea]. Europa Press [consultada 2 de mayo, 2010] Disponible en Internet:http://www.europapress.es/epsocial/ politica-social/noticia-australia-suspendeconcesiones-asilo-afganos-ceilandeses-evitar-trafico-personas-20100409080111.html

PM'S soft boat policy links [en línea]. The Herald Sun [consultado 1 de mayo,2010].Disponible en Internet: http://www.heraldsun. com.au/opinion/editorials/pms-soft-boatpolicy-sinks/story-e6frfhqo-1225852003403

Australia's Refugee and Humanitarian Program: Community views on current challenges and future directions. Refugee Council of Australia, 2009.81 p.

Tony Abbott mocks government suspension of asylum seeker claims [en línea].The Herald Sun [consultado 1 de mayo, 2010]. Disponible en Internet: http://www.heraldsun. com.au/news/national/tony-abbott-mocksgovernment-suspension-of-asylum-seekerclaims/story-e6frf7l6-1225852025577

Refugee Convention,Article 1A(2), Protocol, Art 1(A) (2).Citado por: Refugee and Humanitarian Program. Asa Group [consultado 1 de mayo, 2010]. Disponible en Internet: http://www.australia-migration.com/page/ Refugee_and_Humanitarian_Program/34

National Communications Branch, Department of Immigration and Citizenship, Canberra. Fact Sheet 60 - Australia's Refugee and Humanitarian Program [en línea] [Consultado 1 de mayo,2010] Disponible en Internet: www.immi.gov.au

Asia Pacific Migration Research Network (APMRN). Migration issues in the Asia Pacific: Issues paper from Australia [en línea]. [Consultado 13 de febrero, 2010] Disponible en Internet: http://www.unesco.org/most/ apmrnwp5.htm

Australia asylum suspension could harm world's most vulnerable [en línea].Amnesty International [consultado 1 de mayo, 2010] Disponible en Internet: http://www.amnesty.org/en/news-and-updates/australiaasylum-suspension-could-harm-worldsmost-vulnerable-2010-04-09

Halt in refugee processing creates detention concerns [en línea]. The Refugee Council of Australia [consultado 3 de mayo,2010].Disponible en Internet: http:// www.refugeecouncil.org.au/docs/releases/2010/100418\%20reopen\%20curtin\%20 a\%20step\%20backwards.pdf

UNHCR Review-Sri Lanka Is unsafe: Refugee groups call on the government to unfreeze Sri Lankan asylum claims [en línea]. Refugee Action Coalition Sydney [consultado 3 de mayo,2010] Disponible en Internet: http:// refugeeactioncoalitionsydney.wordpress. com/2010/04/28/unhcr-review\%e2\%80\%93sri-lanka-is-unsafe-refugee-groups-call-onthe-government-to-unfreeze-sri-lankanasylum-claims/ 